Artículo científico

Volumen 30(3):751-765. Septiembre-diciembre, 2019

e-ISSN 2215-3608, doi:10.15517/am.v30i3.36296

http://www.revistas.ucr.ac.cr/index.php/agromeso

\title{
Estrés calórico y conteo de dípteros hematófagos en sistemas silvopastoriles de estratos múltiples ${ }^{1}$
}

\section{Heat stress and blood-sucking diptera count in multi-level silvopastoral systems}

\author{
Wilson Andrés Barragán-Hernández ${ }^{2}$, Juan Carlos Benavidez-Cruz ${ }^{3}$,Aldemar Zúñiga-López $z^{3}$, \\ Amado Espitia-Pacheco ${ }^{4}$, Jaime Cardozo-Cerquera ${ }^{3}$
}

1 Recepción: 21 de febrero, 2019. Aceptación: 15 de mayo, 2019. Este trabajo formó parte de la investigación: "Recomendaciones tecnológicas, socioeconómicas y ambientales para contribuir a la adopción de sistemas agrosilvopastoriles en el Caribe Húmedo", financiado y ejecutado por la Corporación Colombiana de Investigación Agropecuaria (Agrosavia), COL.

2 Corporación Colombiana de Investigación Agropecuaria (Agrosavia), Centro de Investigación Turipaná. Cereté, Colombia. wbarraganh@ agrosavia.co (autor para correspondencia, http://orcid.org/0000-0003-3528-4296).

3 Corporación Colombiana de Investigación Agropecuaria (Agrosavia), Centro de Investigación Tibaitatá. Mosquera, Colombia. jbenavidez@agrosavia.co, azuniga@agrosavia.co,jcardozo@agrosavia.co

4 Universidad de Sucre, Sincelejo, Colombia. amado.espitia@unisucre.edu.co

\section{Resumen}

Introducción. Los sistemas silvopastoriles son esquemas de producción ganadera con potencial para mitigar el efecto negativo del ambiente en la productividad animal. Objetivo. El objetivo del presente trabajo fue evaluar los efectos de sistemas silvopastoriles de estratos múltiples sobre el estrés calórico y la población de dípteros hematófagos en ganado de carne. Materiales y métodos. Se analizó entre mayo y octubre del 2014, en el centro de investigación Turipaná, Córdoba, Colombia, el comportamiento de animales Cebú Brahman y Romosinuano, en dos arreglos silvopastoriles (aSSP) con diversidad en su composición y estratificación vegetal (p-Arbust con dos estratos y p-Arbust-Arbor con tres estratos) y en una pradera sin árboles, con un diseño de bloques completos al azar. En cada tratamiento se evaluaron la temperatura ambiental (TA) y la humedad relativa (HR), y se construyó un índice de temperatura-humedad (ITH). En los animales se evaluó la temperatura de piel (TP) y la frecuencia respiratoria (FR) a dos horas del día (6:00 y 13:00), y se contaron los dípteros hematófagos (CDH) entre las 8:00 y 10:00 h. Los datos se sometieron a un análisis de varianza para modelos mixtos en las variables TA, HR, TP e ITH y modelos mixtos generalizados para CDH y FR. Resultados. Las variables ambientales estuvieron influenciadas por múltiples factores, entre ellos, el cambio en los horarios de muestreo y el arreglo silvopastoril. La mayor carga ambiental (ITH) se observó durante la tarde, evaluación que influyó significativamente sobre la respuesta en FR con aumentos que alcanzaron 2,1 veces más en el tratamiento Pasto, contra 0,8 en p-Arbust-Arbor. Conclusiones. Los aSSP evaluados presentaron ventajas comparativas frente a la reducción del estrés calórico y el conteo de moscas hematófagas bajo condiciones del valle medio del río Sinú. El aSSP p-Arbust, logró mantener los conteos de moscas más bajos a través del tiempo.

Palabras clave: agroforestería, ectoparásitos, tasa de respiración, factores climáticos, cebú, animales de carne. 


\begin{abstract}
Introduction. Silvopastoral systems are livestock scheme production schemes with the potential to mitigate the negative effect of the environment on animal productivity. Objective. The objective of this work was to evaluate the effects of multi-stratum silvopastoral systems on heat stress and the population of hematophagous dipterans in beef cattle. Materials and methods. Between May and October 2014, in the Turipaná research center in Córdoba, Colombia, the behavior of Cebu Brahman and Romosinuano animals was analyzed in two silvopastoral arrangements (aSSP) with diversity in their composition and plant stratification (p-Arbust with two strata and p-Arbust-Arbor with three strata) and in a treeless prairie, with a random complete block design. In each treatment, the environmental temperature (TA) and relative humidity ( $\mathrm{RH})$ were evaluated, and a temperature-humidity index (ITH) was constructed. In animals the skin temperature (TP) and respiratory rate (FR) were evaluated at two hours of the day (6:00 and 13:00), and hematophagous diptera (CDH) were counted between 8:00 and 10:00 a.m. The data were submitted to a variance analysis for mixed models in the variables TA, HR, TP and ITH and generalized mixed models for CDH and FR. Results. The environmental variables were influenced by multiple factors including: the change in sampling schedules, and the silvopastoral arrangement. The highest environmental load (ITH) was observed during the afternoon, an evaluation that significantly influenced the response in FR with increases that reached 2.1 times more in the Pasto treatment, against 0.8 in p-Arbust-Arbor. Conclusions. The aSSP evaluated presented comparative advantages against the reduction of heat stress and the count of hematophage flies under conditions of the middle valley of the Sinu River. The aSSP p-Arbust managed to keep fly counts lower over time.
\end{abstract}

Keywords: agroforestry, ectoparasites, respiration rate, climatic factors, zebu, meat animals.

\title{
Introducción
}

Los sistemas de producción pecuarios cubren un rol importante como soporte alimenticio de la población humana, al punto de considerarlos responsables de gran parte del abastecimiento proteico y calórico de la población mundial (Herrero et al., 2014; Weindl et al., 2015). Sin embargo, frente al cambio y la variabilidad climática, son considerados vulnerables, con serias limitantes para su desarrollo y sostenibilidad a corto plazo (Pankaj et al., 2013).

$\mathrm{El}$ incremento de la temperatura y la variación en las precipitaciones, implican un efecto directo e indirecto sobre la productividad de los sistemas pecuarios, asociado entre otros, al estrés calórico, la explosión poblacional de vectores de enfermedades y a la disminución de la capacidad productiva en los animales (Nardone et al., 2010; Pankaj et al., 2013).

Bajo condiciones de estrés térmico una de las principales limitantes de los sistemas pecuarios en ambientes tropicales, la temperatura ambiental induce cambios fisiológicos, metabólicos y conductuales en los animales, que resultan en incrementos de la frecuencia respiratoria, la temperatura corporal y de la piel (Robertshaw, 2006; Arias et al., 2008), alteraciones en el metabolismo ácido-básico (Barragán-Hernández et al., 2015), disminución en el consumo de materia seca, incremento en consumo de agua (Ahlberg et al., 2018) y desarrollo de conductas de búsqueda de sombra e inactividad (Pérez et al., 2008).

Varios autores han documentado (Kadzere et al., 2002; Molina-Benavides et al., 2018) que el efecto negativo del ambiente, representado en el índice de temperatura - humedad (ITH), desencadena respuestas fisiológicas en los bovinos con el objeto de mantener su homeóstasis térmica, entre las cuales, la frecuencia respiratoria (FR) se considera la más importante por su fidelidad con la carga calórica en el animal (Galán et al., 2018). Collier et al. (2011), indicaron que por cada aumento unitario de ITH vacas Holstein incrementaron su FR en 2 rpm. Por su parte, Atkins et al. (2018) en la misma raza, registraron un incremento de 1,9 rpm por debajo de 70 puntos de ITH y $3,1 \mathrm{rpm}$ con ITH>70 puntos. 
Con relación al cálculo de ITH, es importante considerar que la mayoría de ecuaciones propuestas en la literatura no son relevantes para condiciones tropicales (Lallo et al., 2018). Adicionalmente, Atkins et al. (2018) afirmaron que los índices propuestos en la literatura presentan variabilidad en su respuesta bajo diferentes condiciones, lo cual se relaciona con ausencia de factores propios del animal tales como raza, edad, producción, entre otros; y a su exclusiva construcción con base en variables ambientales.

Con relación al estrés por calor, se ha demostrado que la respuesta fisiológica puede inducir inmunodeficiencia en los animales (Hansen, 2004; Nardone et al., 2010), con una consecuente vulnerabilidad a los vectores de enfermedades (Kadzere et al., 2002), factor que se acentúa con el hecho de que los cambios repentinos en la temperatura y humedad ambiental, favorecen la explosión poblacional de vectores, debido a su mayor actividad en ambientes más calurosos (Grace et al., 2015).

La respuesta más probable frente a la explosión poblacional de vectores de enfermedades, aunque poco sostenible y potencialmente peligrosa para el animal, para la salud humana y para el ambiente, es el uso de productos químicos tipo antihelmínticos y acaricidas, entre otros (Alonso-Díaz et al., 2014), de los cuales se ha demostrado que su mal uso, ha generado consecuencias como la resistencia a ecto y endoparásitos (Alegría-López et al., 2015) y alteraciones en la población de invertebrados benéficos del suelo (Alonso-Díaz et al., 2014; Pulido et al., 2018).

El incremento e intensificación en los sistemas de producción que demanda la creciente población humana, requiere un enfoque sostenible, con beneficios en el ecosistema y rentabilidad económica y social, basado en sistemas con uso de pasturas y forrajes (Rao et al., 2015). Los sistemas silvopastoriles han sido considerados como herramientas promisorias ante el cambio y la variabilidad climática (Murgueitio-Restrepo et al., 2016), con ventajas comparativas frente a esquemas de producción convencionales en aspectos relacionados con el bienestar animal (Broom, 2017), e interacciones ecológicas que favorecen el control natural de plagas y vectores de enfermedades (Giraldo et al., 2011).

El objetivo del presente trabajo fue evaluar los efectos de los sistemas silvopastoriles de estratos múltiples sobre el estrés calórico y la población de dípteros hematófagos en ganado de carne.

\section{Materiales y métodos}

\section{Área de estudio y diseño del experimento}

El estudio se desarrolló en el Centro de Investigación Turipaná, perteneciente a la Corporación Colombiana de Investigación Agropecuaria (AGROSAVIA), con sede en Cereté (Córdoba, Colombia. 0851' N, 7549' O, altitud de 18 m), ubicado en la Región Caribe colombiana. Esta región presenta dos períodos climáticos, uno de lluvias entre los meses de mayo y noviembre y otro seco de diciembre a abril. La precipitación promedio anual es de $1380 \mathrm{~mm}$, con una temperatura media anual de $28^{\circ} \mathrm{C}$. Durante el año se registra una evapotranspiración potencial de $1240 \mathrm{~mm}$ y humedad relativa de $81 \%$ (datos de la estación climatológica Agrosavia-Turipaná). Según la clasificación de zonas de vida (Holdridge, 1971), el área se clasifica ecológicamente como bosque seco tropical (bs-T).

Se evaluaron dos arreglos silvopastoriles y una pradera sin árboles, pertenecientes a un experimento establecido en el año 1998, el cual se desarrolló para establecer arreglos silvopastoriles con base en el conocimiento local de ganaderos sobre especies arbustivas, arbóreas y maderables de interés pecuario en los departamentos de Córdoba, Bolívar, Sucre y Atlántico de la Región Caribe colombiana (Cajas-Giron y Sinclair, 2001).

Los arreglos silvopastoriles (aSSP) evaluados presentaron tres estratos distribuidos en especies herbáceas, arbustivas y arbóreas (árboles productores de frutos). El tratamiento sin árboles (Pasto) estuvo conformado por las especies herbáceas (Poaceae), en orden de importancia: Megathyrsus maximus (Jacq.) B.K. Simon \& S.W.L. 
Jacobs, Dichanthium aristatum (Poir.) C.E. Hubb y Cynodon nlemfuensis Vanderyst. El primer aSSP (p-Arbust) presentó dos estratos, un estrato herbáceo, conformado por las gramíneas mencionadas y otro por los arbustos Leucaena leucocephala (Lam.) de Wit. y Crecentia cujete L. (como arbusto productor de forraje y no de frutos), y el segundo arreglo (p-Arbust-Arbor) el cual presentó tres estratos, el primero conformado principalmente por la gramínea M. maximus, el segundo por los arbustos L. leucocephala y C. cujete, y el tercero, estrato arbóreo, por Cassia grandis L.F, Pithecellobium saman (Jacq.) Benth. y Guazuma ulmifolia Lam. (Cuadro 1).

Cuadro 1. Composición botánica y densidades de siembra de los arreglos silvopastoriles evaluados y de la pradera sin árboles. Centro de Investigación Turipaná. Cereté, Córdoba, Colombia. 2014

Table 1. Botanical composition and planting densities of the evaluated silvopastoral arrangements and the treeless meadow. Turipaná Research Center, Cereté, Cordoba, Colombia. 2014.

\begin{tabular}{|c|c|c|c|}
\hline Tratamiento & Descripción & Estratos & Especies (\% composición herbácea) \\
\hline Pasto & Pastura & 1 & $\begin{array}{c}\text { Estrato herbáceo = Megathyrsus maximus }(40 \%) \text {, Dichanthium } \\
\text { aristatum }(34 \%) \text {, Cynodon nlemfuensis }(13 \%) \text {, otras especies } \\
(5 \%) \text { y leguminosas herbáceas }(8 \%) .\end{array}$ \\
\hline p-Arbust & $\begin{array}{l}\text { Pastura }+ \text { arbustos } \\
\text { productores de } \\
\text { hojas }\end{array}$ & 2 & $\begin{array}{c}\text { Estrato herbáceo = Megathyrsus maximus }(49 \%) \text {, Dichanthium } \\
\text { aristatum }(25 \%) \text {, Cynodon nlemfuensis }(10 \%) \text {, otras especies } \\
(10 \%) \text { y leguminosas herbáceas }(6 \%) . \\
\text { Estrato arbustivo = Leucaena leucocephala y Crescentia cujete. } \\
\text { Densidad de arbustos }=625 \text { árboles ha }{ }^{-1} . \\
\text { Distancia de siembra } 4 \mathrm{~m} \mathrm{x} 4 \mathrm{~m} .\end{array}$ \\
\hline p-Arbust-Arbor & $\begin{array}{l}\text { Pasto }+ \text { arbustos } \\
\quad+\text { árboles } \\
\text { (produductores de } \\
\quad \text { frutos) }\end{array}$ & 3 & 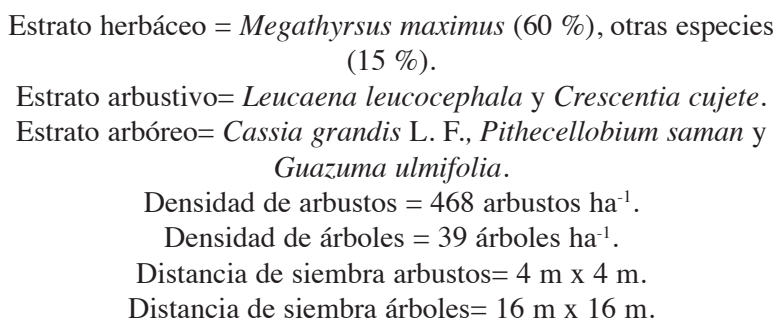 \\
\hline
\end{tabular}

Fuente: adaptado de Barragán-Hernández et al. (2015) / Source: adapted from Barragán-Hernández et al. (2015).

Los tratamientos evaluados (arreglos silvopastoriles y pradera sin árboles) se establecieron bajo un diseño de bloques al azar con tres repeticiones (tres bloques por condición de drenaje en suelo: deficiente, moderado y óptimo). Cada unidad experimental estuvo constituida por dos hectáreas (100 m de ancho x $200 \mathrm{~m}$ de largo), divididas en ocho potreros de $2500 \mathrm{~m}^{2}$ cada uno, los cuales se manejaron bajo un esquema de rotación de tres días de ocupación y veintiún días de descanso.

\section{Animales experimentales y variables evaluadas}

En cada tratamiento se asignaron de manera aleatoria tres bovinos machos de raza Romosinuano y tres de raza Cebú Brahman. Los animales Romosinuano provenían del banco de germoplasma del Instituto Colombiano Agropecuario, bajo custodia de AGROSAVIA en el C.I. Turipaná (Ossa-Saraz et al., 2013), con un peso de $260 \pm 22,3 \mathrm{~kg}$ y 18 meses de edad; mientras que, los Brahman con $218 \pm 29 \mathrm{~kg}$ con 13 meses de edad, prevenientes de un lote comercial de propiedad del C.I. Turipaná. Los animales se manejaron bajo pastoreo rotacional, con suministro de sal mineralizada y agua a voluntad. 
Las mediciones se llevaron a cabo durante los meses de mayo a octubre del año 2014, los martes y jueves de cada semana, a las 6:00 h y 13:00 h del día. Se evaluó la temperatura ambiental (TA) y la humedad relativa (HR), mediante un termohigrómetro digital tipo USB. En los animales, la frecuencia respiratoria (FR) se midió contando ininterrumpidamente los movimientos del flanco en cada animal (Gaughan et al., 2000), representada en respiraciones por minuto (rpm). La temperatura de la piel (TP) se obtuvo con un termómetro infrarrojo laser $( \pm 2,5$ ${ }^{\circ} \mathrm{C}$ ), colocado a una distancia de 1,5 m del animal; se tomaron tres lecturas en cada animal (cuello, espalda y anca), las cuales se promediaron para el análisis de los datos.

El conteo de dípteros hematófagos la realizaron evaluadores entrenados, entre las 8:00h y 10:00h de la mañana, por un costado del animal en pie (Castro et al., 2005; Oliveira et al., 2015). Para efectos del análisis, el número de dípteros totales se obtuvo multiplicando el conteo en un costado del animal por dos (Lima et al., 2003).

Con las variables TA y HR, se construyó el índice de temperatura-humedad (ITH), según lo descrito por Dikmen y Hansen (2009).

\section{Análisis de la información}

Los datos obtenidos se registraron y almacenaron en hojas de cálculo, para posteriormente analizarlos en el software estadístico R-Project; con el empleo de las librerías lmer (Bates et al., 2015) para modelos mixtos, se estudiaron las variables TA, HR, TP. Además, se usó la función glmmTBM (Brooks et al., 2017) para modelos mixtos generalizados en el análisis del conteo de dípteros hematófagos y FR, con distribución de residuales binomial negativa y función de enlace $\log$, con ajuste de modelo inflado por ceros para conteo de dípteros hematófagos y truncado en ceros para frecuencia respiratoria. En ambos casos, se siguieron los lineamientos propuestos por Zuur et al. (2009). En las variables ambientales, se consideró como efecto fijo el aSSP (Pasto, p-Arbust y p-ArbustArbor), la hora (6 y 13 h) y la fecha (de mayo a octubre), así como la interacción de estos factores. Como efectos aleatorios se consideraron el bloque (deficiente, moderado y óptimo), el evaluador (1 y 2) y el error de la medida repetida en el tiempo.

Para las variables evaluadas en los animales, en los efectos fijos además de los mencionados anteriormente se incluyó el efecto de la raza (Romosinuano y Brahman). En todos los casos, bajo un modelo saturado y el uso del estimador de máxima verosimilitud restringida, se evaluó la pertinencia del efecto aleatorio y de la modelación de estructuras de varianzas y covarianzas para las medidas repetidas en el tiempo, mediante los criterios de información (datos no presentados) AIC y BIC (Di-Rienzo et al., 2009; Cayuela, 2012).

\section{Resultados}

El análisis de varianza indicó efecto $(\mathrm{p}<0,05)$ del aSSP, la hora, el mes de evaluación y las interacciones aSSP:hora, aSSP:mes y hora:mes, sobre las variables TA, HR e ITH (Cuadro 2). Para las variables evaluadas en el animal, la FR presentó efecto $(\mathrm{p}<0,05)$ de todos los factores principales e interacciones evaluadas, con excepción $(\mathrm{p}>0,05)$ de las interacciones aSSP:mes, aSSP:raza:mes, raza:hora:mes y aSSP:raza:hora:mes. Para el caso de la TP, se reportó diferencia $(\mathrm{p}<0,05)$ en los efectos principales hora y mes, y en las interacciones aSSP:raza, hora:mes y aSSP:raza:mes. El conteo total de dípteros hematófagos en el animal registró efecto $(p<0,05)$ de los factores principales raza y mes, con una tendencia estadística en el efecto del aSSP ( $\mathrm{p}=0,0503)$; en esta variable, se registró efecto en las interacciones $(\mathrm{p}<0,05)$ aSSP:mes, raza:mes y aSSP:raza:mes.

Los efectos principales no fueron analizados de manera aislada por haber sido afectados por interacciones. Por consiguiente, se discutieron las interacciones de más alto orden y de carácter significativo reportadas para las variables evaluadas. 
Cuadro 2. Variables ambientales, respuesta fisiológica al estrés calórico y conteo de dípteros hematófagos para dos arreglos silvopastoriles y una pradera sin árboles. C.I. Turipaná, Cereté, Córdoba, Colombia. Mayo - octubre 2014

Table 2. Environmental variables, physiological response to heat stress and hematophagus diptera count for two silvopastoral arrangements and treeles meadow. C.I. Turipana, Cerete, Cordoba, Colombia. May - Octuber 2014.

\begin{tabular}{|c|c|c|c|c|c|c|}
\hline & $\mathbf{T A}^{1}$ & HR & ITH $^{1}$ & FR & $\mathbf{T} \mathbf{P}^{1}$ & DP \\
\hline & \multicolumn{6}{|c|}{ Arreglo silvopastoril (aSSP) } \\
\hline Pasto & $31,91 \mathrm{a}$ & 69,82 a & 82,92 b & $47,47 \mathrm{~b}$ & 36,99 & 55,17 \\
\hline p-Arbust & $31,71 \mathrm{a}$ & $71,01 \mathrm{a}$ & $82,75 \mathrm{ab}$ & $49,99 \mathrm{c}$ & 36,95 & 36,39 \\
\hline \multirow[t]{2}{*}{ p-Arbust-Arbor } & $30,21 \mathrm{~b}$ & $74,49 \mathrm{~b}$ & $81,42 \mathrm{a}$ & $41,66 \mathrm{a}$ & 35,76 & 43,49 \\
\hline & \multicolumn{6}{|c|}{ Hora $(\mathbf{H})$} \\
\hline $6: 00 \mathrm{~h}$ & $26,01 \mathrm{~b}$ & $92,49 \mathrm{~b}$ & 77,88 a & $30,26 \mathrm{a}$ & $33,80 \mathrm{a}$ & - \\
\hline \multirow[t]{2}{*}{$13: 00 \mathrm{~h}$} & $36,53 \mathrm{a}$ & $51,06 \mathrm{a}$ & $86,84 \mathrm{~b}$ & $70,66 \mathrm{~b}$ & $39,33 \mathrm{~b}$ & - \\
\hline & \multicolumn{6}{|c|}{$\operatorname{Raza}(\mathbf{R})$} \\
\hline Romosinuano & - & - & - & $53,62 \mathrm{~b}$ & 36,66 & $57,2 \mathrm{~b}$ \\
\hline Brahman & - & - & - & $39,87 \mathrm{a}$ & 36,47 & $34,9 \mathrm{a}$ \\
\hline \multicolumn{7}{|l|}{ Mes (M) } \\
\hline Mayo & $31,71 \mathrm{~b}$ & $67,03 \mathrm{a}$ & $82,53 \mathrm{~b}$ & $41,5 \mathrm{a}$ & $35,70 \mathrm{a}$ & $130,50 \mathrm{c}$ \\
\hline Junio & $32,73 \mathrm{c}$ & 67,11 a & $83,61 \mathrm{c}$ & $47,57 \mathrm{bc}$ & $36,82 \mathrm{~b}$ & $50,94 \mathrm{~b}$ \\
\hline Julio & $32,80 \mathrm{c}$ & 67,69 a & $83,77 \mathrm{c}$ & $52,31 \mathrm{~d}$ & $37,39 \mathrm{~b}$ & 25,13 a \\
\hline Agosto & $31,23 \mathrm{~b}$ & $73,11 \mathrm{~b}$ & $82,68 \mathrm{~b}$ & $48,40 \mathrm{c}$ & $36,84 \mathrm{ab}$ & $46,23 \mathrm{~b}$ \\
\hline Septiembre & $29,48 \mathrm{a}$ & $77,11 \mathrm{~b}$ & $80,53 \mathrm{a}$ & $43,92 \mathrm{ab}$ & $36,31 \mathrm{ab}$ & $44,98 \mathrm{~b}$ \\
\hline Octubre & $29,71 \mathrm{a}$ & $78,62 \mathrm{~b}$ & $81,05 \mathrm{a}$ & $44,51 \mathrm{ab}$ & $36,34 \mathrm{ab}$ & $22,06 \mathrm{a}$ \\
\hline \multicolumn{7}{|l|}{ Valor de $p$} \\
\hline aSSP & 0,0003 & 0,008 & 0,031 & $<0,001$ & 0,0748 & 0,0503 \\
\hline Raza & - & - & - & $<0,001$ & 0,0821 & $<0,001$ \\
\hline Hora & $<0,001$ & $<0,001$ & $<0,001$ & $<0,001$ & $<0,001$ & - \\
\hline Mes & $<0,001$ & $<0,001$ & $<0,001$ & $<0,001$ & 0,010 & $<0,001$ \\
\hline aSSP:R & - & - & - & $<0,001$ & 0,0199 & 0,0968 \\
\hline aSSP:H & $<0,001$ & 0,0182 & 0,0028 & $<0,001$ & $<0,001$ & - \\
\hline aSSP:M & 0,9642 & 0,6699 & 0,7951 & 0,0781 & 0,9511 & 0,015 \\
\hline $\mathrm{R}: \mathrm{H}$ & - & - & - & $<0,001$ & 0,8096 & - \\
\hline $\mathrm{R}: \mathrm{M}$ & - & - & - & 0,0122 & 0,2445 & 0,0105 \\
\hline $\mathrm{H}: \mathrm{M}$ & $<0,001$ & $<0,001$ & $<0,001$ & $<0,001$ & 0,0002 & - \\
\hline aSSP:R:H & - & - & - & 0,00803 & 0,3152 & - \\
\hline aSSP:R:M & - & - & - & 0,66095 & 0,0032 & $<0,001$ \\
\hline aSSP:H:M & 0,5554 & 0,7477 & 0,4093 & $<0,001$ & 0,0833 & - \\
\hline R:H:M & - & - & - & 0,24177 & 0,0416 & - \\
\hline aSSP:R:H:M & - & - & - & 0,09591 & 0,3954 & - \\
\hline
\end{tabular}

${ }^{1}$ Estructura de varianza constante en los factores hora y aSSP / Constant variance in factors hour and aSSP.

TA: temperatura ambiental; HR: humedad relativa; ITH: índice de temperatura y humedad; FR: frecuencia respiratoria; TP: temperatura de piel. DP: díptero hematófago / TA: air temperature; RH: relative humidity; THI: temperature-humidity index; FR: respiration rate; ST: skin temperature DP: hematophagous diptera.

p-Arbust: sistema silvopastoril de dos estratos. Estrato herbáceo: M. maximus, D. aristatum y C. nlemfuensis. Estrato arbustivo: L. leucocephala y C. cujete / p-Arbust: two level silvopastoral systems. Herbaceous level: M. maximus D. aristatum and C. nlemfuensis. Shrub level: L. leucocephala and C. cujete.

p-Arbust-Arbor: sistema silvopastoril de tres estratos. Estrato herbáceo: M. maximus D. aristatum y C. nlemfuensis. Estrato arbustivo: L. leucocephala y C. cujete. Estrato tres: C. grandis, P. saman y G. ulmifolia / p-Arbust-Arbor: Three level Silvopastoral systems. Herbaceous level: M. maximus D. aristatum and C. nlemfuensis. Shrub level: L. leucocephala and C. cujete. Tree level: C. grandis, P. saman y G. ulmifolia. 
En las variables ambientales, la HR fue afectada por las interacciones con los factores hora y mes. Para el caso de la interacción con el factor hora, los valores medios de HR a las 6:00h oscilaron entre 90,6 \% (Pasto) y 94,27 \% ( $\mathrm{p}$-Arbust-Arbor), sin diferencia entre ellos ( $\mathrm{p}>0,05)$, pero estadísticamente diferentes a los observados a las 13:00 $\mathrm{h}$ en el arreglo p-Arbust-Arbor (54,71\%) y con p-Arbust $(49,48 \%)$ y Pasto $(48,99 \%)$, estos últimos sin diferencias $(\mathrm{p}>0,05)$. La TA registró el mismo comportamiento evidenciado en la HR, sin diferencia $(\mathrm{p}>0,05)$ a las 6:00 h (varió entre 26,3 y $\left.25,5^{\circ} \mathrm{C}\right)$. Contrario a esto, a las $13: 00 \mathrm{~h}$ el aSSP p-Arbust-Arbor registró la menor TA $\left(34,75^{\circ} \mathrm{C}\right)$, con diferencia $(\mathrm{p}<0,05)$ del registro obtenido para los tratamientos Pasto y p-Arbust, en los cuales se observó un valor medio de 37,47 y $37,27^{\circ} \mathrm{C}$ sin diferencia entre ellos ( $\left.>>0,05\right)$. Por último, el ITH presentó diferencias $(\mathrm{p}<0,05)$ entre los valores evaluados a las 6:00 h y 13:00 h en cada aSSP, con los valores más bajos obtenidos en el aSSP p-ArbusArbor del orden de 77,36 \% y 85,84 \% para la evaluación en la mañana y en la tarde, respectivamente. En las tres variables de orden ambiental, las evaluaciones obtenidas a las 6:00 h y 13:00 h difirieron entre sí $(\mathrm{p}<0,05)$ a través de los meses evaluados (Figura 1).

Para el caso de la temperatura de la piel (TP) en la interacción aSSP, raza y mes de evaluación, solo se registró diferencia $(\mathrm{p}<0,05)$ entre los valores observados en las razas Romosinuano $\left(31,1{ }^{\circ} \mathrm{C}\right)$ y Cebú Brahman $\left(31,2{ }^{\circ} \mathrm{C}\right)$ en el aSSP p-Arbust-Arbor, con el registro obtenido en la raza Romosinuano para el mes de julio $\left(38,2^{\circ} \mathrm{C}\right)$ en el tratamiento control. Para la interacción raza, hora y mes, las variaciones en la TP estuvieron más influenciadas por la hora que por la raza. Con relación a la FR en la interacción de los factores aSSP, raza y hora, el análisis de varianza no indicó diferencia ( $\mathrm{p}>0,05)$ para la evaluación de las 6:00 h, con tasas de respiración que oscilaron entre 26 y $35 \mathrm{rpm}$, asociadas a condiciones de termoneutralidad en las razas evaluadas (Hammond et al., 1996). Contrario a esto, la evaluación de las 13:00 h evidenció incrementos en la FR, lo cual se asocia a que el aumento de las 6:00 $\mathrm{h}$ a las 13:00 h en el ITH afectó la homeóstasis térmica en los animales, con incremento en la FR como mecanismo para perder calor (Robertshaw, 2006).

Los animales Cebú Brahman y Romosinuano vinculados al aSSP p-Arbust-Arbor, registraron los menores incrementos en la tasa respiratoria con valores de 0,81 y 0,94 veces la respiración observada a las 6:00 h, respectivamente; valores que difirieron $(\mathrm{p}<0,05)$ de los registrados en los tratamientos $\mathrm{p}$-Arbustos con incrementos de 1,46 y 1,70 veces, y Pasto con incrementos de 1,21 y de 2,10 veces, respectivamente, para las razas antes mencionadas.

A través de los meses de evaluación, la FR se mantuvo constante a las 6:00h. Sin embargo, la valoración de las 13:00 h evidenció que el aSSP p-Arbust-Arbor fue el tratamiento en el cual la FR se logró mantener constante y solamente incrementó de manera significativa durante el mes de julio, con valor de $66 \mathrm{rpm}$, sin reportar diferencia ( $>0,05)$ en los demás meses evaluados (con registros entre 48 y $60 \mathrm{rpm}$ ). Para el tratamiento p-Arbust, la FR describió un comportamiento parabólico, el mismo registró la menor FR en los meses de mayo, septiembre y octubre con 66, 71, 69 rpm, respectivamente, y la mayor en los meses de junio, julio y agosto, con 88, 94 y 99 rpm, respectivamente. En el tratamiento Pasto, la menor FR se reportó para el mes de mayo (57 rpm), a partir de la cual aumentó $(\mathrm{p}<0,05)$ a valores que variaron entre 73 y $91 \mathrm{rpm}$ para los meses de junio a octubre (Figura 2).

En el conteo de dípteros hematófagos para animales de ambas razas estudiadas, los tratamientos Pasto y

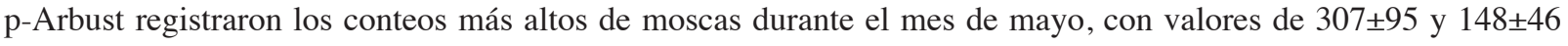
moscas/animal para la raza Cebú Brahman y $138 \pm 38$ y $142 \pm 47$ moscas/animal para la raza Romosinuano, respectivamente. Con el tratamiento Pasto se obtuvieron $126 \pm 70,103 \pm 31$ y $89 \pm 34$ moscas/animal en los meses de agosto, septiembre y octubre, respectivamente; mientras que para p-Arbust-Arbor se reportaron $82 \pm 24,64 \pm 20$ y $39 \pm 28$ moscas/animal en agosto, septiembre y octubre, respectivamente. Independientemente de la raza, se observó que el tratamiento p-Arbust, registró el conteo de dípteros hematófagos más bajos a través del tiempo (Figura 3). 

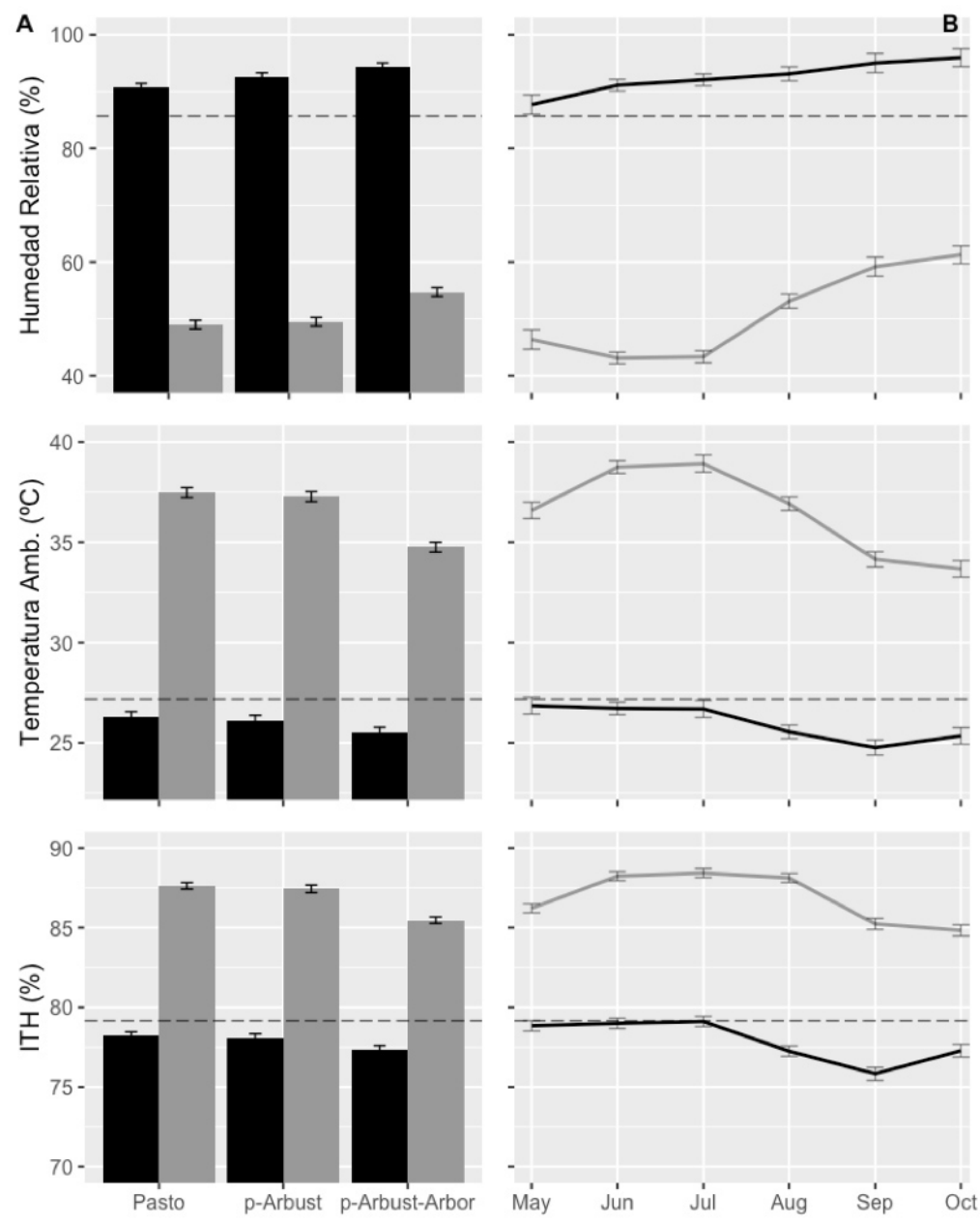

Figura 1. Interacciones arreglos silvopastoriles (aSSP) por hora (A) y hora por mes (B), para las variables temperatura ambiental, humedad relativa e índice de temperatura-humedad, para dos aSSP y una pradera sin árboles. Centro de Investigación Turipaná. Cereté, Córdoba, Colombia. Mayo - octubre. 2014.

Barras sólida y líneas de color negro representan la evaluación a las 6:00 h y color gris a las 13:00 h. La línea de punto representa el intercepto del modelo (media general) para cada variable. ITH: índice de temperatura y humedad.

p-Arbust: sistema silvopastoril de dos estratos. Estrato herbáceo: M. maximus D. aristatum y C. nlemfuensis. Estrato arbustivo: L. leucocephala y $C$. cujete. p-Arbust-Arbor: sistema silvopastoril de tres estratos. Estrato herbáceo: M. maximus D. aristatum y C. nlemfuensis. Estrato arbustivo: L. leucocephala y C. cujete. Estrato tres: C. grandis, P. saman y G. ulmifolia.

Figure 1. Silvopastoral arrangements aSSP interactions per Hour (A), and month per hour (B) for the variables environmental temperature, relative humidity and temperature-humidity index, for two ASSP and treeless meadow. Turipaná Research Center. Cereté, Córdoba, Colombia. May - October. 2014.

Solid bars and black lines represent the evaluation at 6:00 $\mathrm{h}$ and gray color at 13:00 $\mathrm{h}$. The dotted line represents the intercept of the model (overall mean) for each variable. THI: temperature-humidity index.

p-Arbust: Two level silvopastoral systems. Herbaceous level: M. maximus D. aristatum and C. nlemfuensis. Shrub level: L. leucocephala and C. cujete. p-Arbust-Arbor: Three level silvopastoral systems. Herbaceous level: M. maximus D. aristatum and C. nlemfuensis. Shurb level: L. leucocephala and C. cujete. Tree level: C. grandis, P. saman and G. ulmifolia. 

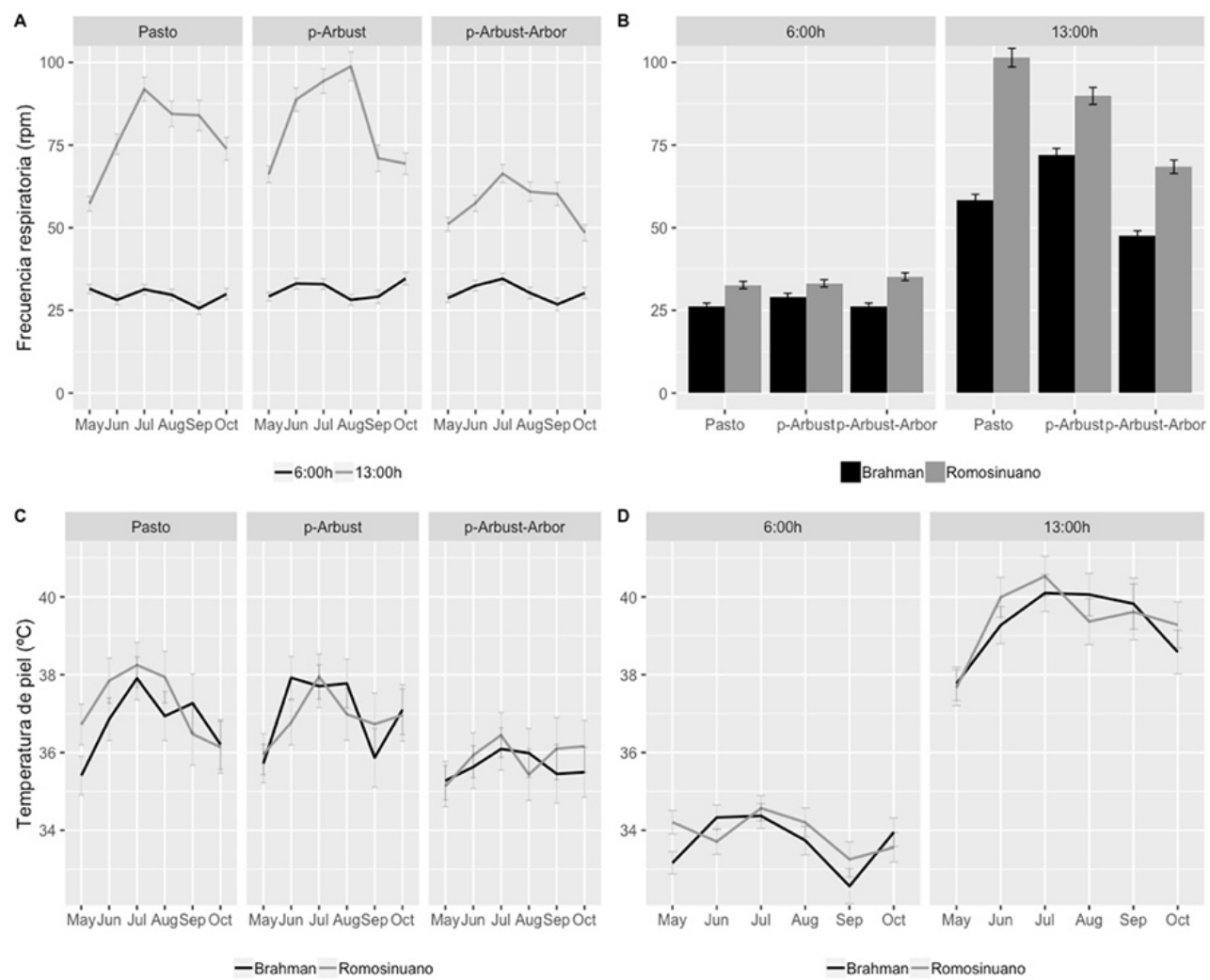

Figura 2. Interacción entre arreglos silvopastoriles (aSSP), hora y mes (A) y aSSP, raza y hora (B), para la frecuencia respiratoria e interacción aSSP, raza y mes (C) y hora, raza (Romosinuano y Cebú Brahman) y mes (D) para la temperatura de la piel en dos aSSP y una pradera sin árboles. Centro de Investigación Turipaná. Cereté, Córdoba, Colombia. Mayo - octubre 2014.

p-Arbust: sistema silvopastoril de dos estratos. Estrato herbáceo: M. maximus, M. maximus, D. aristatum y C. nlemfuensis. Estrato arbustivo: L. leucocephala y $C$. cujete. p-Arbust-Arbor: sistema silvopastoril de tres estratos. Estrato herbáceo: M. maximus, M. maximus, D. aristatum y C. nlemfuensis. Estrato arbustivo: L. leucocephala y C. cujete. Estrato tres: C. grandis, P. saman y G. ulmifolia

Figure 2. Interaction between silvopastoral arrangements (aSSP), hour and month (A) and aSSP, breed and time (B) for respiratory rate and aSSP interaction, breed and month $(\mathrm{C})$ and time, breed (Romosinuano and Cebu Brahman) and month (D) for skin temperature in two aSSP and a meadow without trees. Turipaná Research Center. Cereté, Córdoba, Colombia. May - October 2014.

p-Arbust: two level silvopastoral systems. Herbaceous level: M. maximus, D. aristatum, and C. nlemfuensis. Shrub level: L. leucocephala and C. cujete. p-Arbust-Arbor: Three level Silvopastoral systems. Herbaceous level: M. maximus, D. aristatum, and C. nlemfuensis. Shurb level: L. leucocephala and C. cujete. Tree level: C. grandis, P. saman and G. ulmifolia.

\section{Discusión}

En el presente estudio, se observó el efecto negativo del ambiente al relacionar el incremento de la FR con el aumento de ITH durante el día. En la evaluación de las 6:00 h, los animales estuvieron en confort térmico $(<60$ rpm) (Arias et al., 2008; Galán et al., 2018). Sin embargo, a las 13:00 h se observó un incremento en la FR de hasta $201 \%$ veces el número de rpm comparada con la evaluación a las 6:00 h, especialmente en el tratamiento Pasto. 

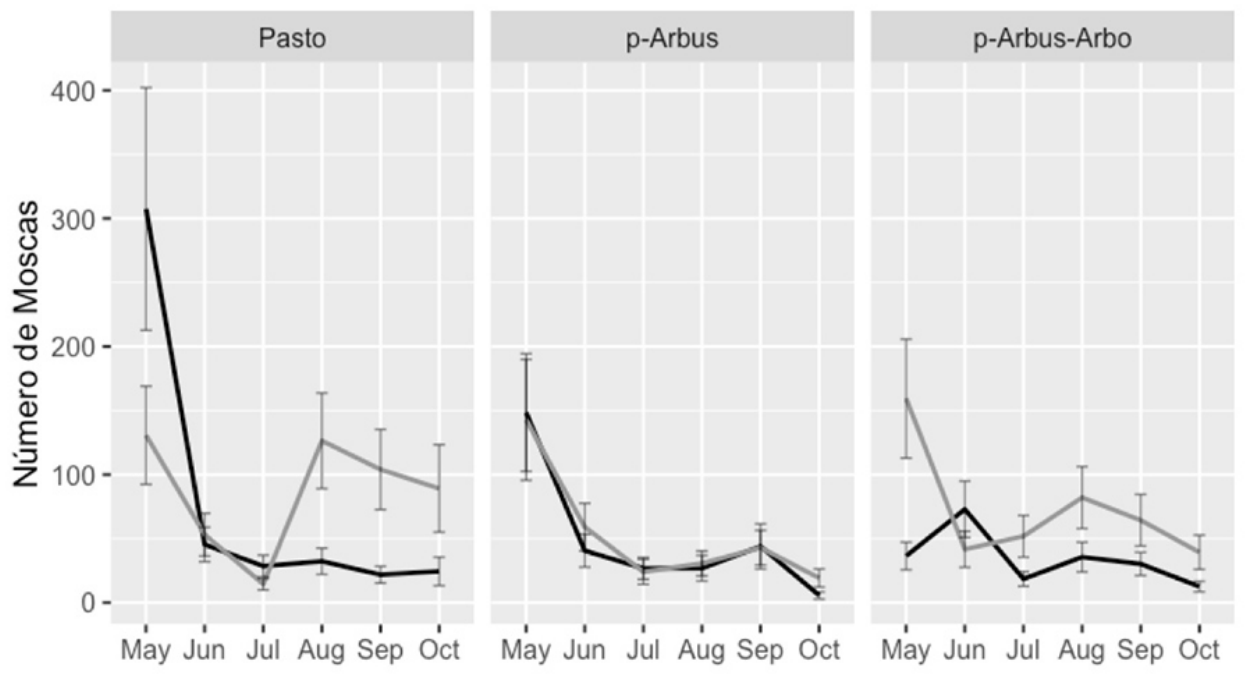

- Brahaman - Romosinuano

Figura 3. Conteo de moscas hematófagas entre los meses de mayo a octubre en las razas Romosinuano y Cebú Brahman para dos arreglos silvopastoriles y una pradera sin árboles. Centro de Investigación Turipaná. Cereté, Córdoba, Colombia. 2014.

p-Arbust: sistema silvopastoril de dos estratos. Estrato herbáceo: M. maximus, D. aristatum y C. nlemfuensis. Estrato arbustivo: L. leucocephala у C. cujete. p-Arbust-Arbor: sistema silvopastoril de tres estratos. Estrato herbáceo: M. maximus, D. aristatum y $C$. nlemfuensis. Estrato arbustivo: L. leucocephala y C. cujete. Estrato tres: C. grandis, P. saman y G. ulmifolia

Figure 3. Fly hematophagous count between the months of May to October in the Romosinuano and Zebu Brahman breed for two silvopastoral arrangement and treeless meadow. Turipaná Research Center. Cereté, Córdoba, Colombia. 2014.

p-Arbust: Two level silvopastoral systems. Herbaceous level: M. maximus, D. aristatum and C. nlemfuensis. Shrub level: L. leucocephala, and C. cujete. p-Arbust-Arbor: three level silvopastoral systems. Herbaceous level: M. maximus, D. aristatum and $C$. nlemfuensis. Shrub level: L. leucocephala, and C. cujete. Tree level: C. grandis, P. saman and G. ulmifolia.

El índice de temperatura y humedad (ITH), se toma como referencia al ser considerado una herramienta que evalúa el impacto conjunto de la temperatura y la humedad en la homeóstasis térmica del animal (Dikmen y Hanse, 2009; Chang-Fung-Martel et al., 2017). Sin embargo, bajo las condiciones para las cuales se desarrolló la presente investigación, los valores de referencia en ITH no se ajustan $(<72$ ITH), dado que las ecuaciones, en su gran mayoría, han sido desarrolladas para animales Bos taurus bajo latitudes diferentes.

Con base en lo descrito por Lallo et al. (2018), quienes han propuesto que para rumiantes en el trópico, el límite máximo del ITH debe ser 84 puntos, se concluyó que en las horas de la tarde todos los bovinos evaluados en la presente investigación, pudieron estar fuera de su zona de confort térmico, dado que en todos los tratamientos evaluados el ITH estuvo por encima de 85 puntos. Sin embargo, al considerar la respuesta en la FR, se pudo observar que tanto los animales Romosinuano como los Cebú Brahman en el aSSP p-Arbust-Arbor (85,4 ITH), mantuvieron su FR por debajo de $60 \mathrm{rpm}$ en la mayoría de los meses evaluados, lo que pude indicar que, si bien podía haber un ambiente hostil, la cobertura de los árboles y el microclima generado en el aSSP logró mitigar el impacto ambiental sobre el estrés calórico en los animales.

Varios autores han documentado el beneficio de la sombra artificial (Schütz et al., 2008; Tucker et al., 2008) y proveniente de árboles (Oliveira et al., 2017), sobre la disminución de los índices climáticos relacionados con el 
estrés calórico. Bajo la sombra de árboles, se han referenciado disminuciones en los índices de estrés térmico del orden de 4 puntos de ITH en la mañana y de 3 por la tarde, al compararlos con condiciones desprovistas de sombra (Lopes et al., 2016). En el presente trabajo se obtuvo una reducción de ITH de 2,1 puntos al comparar a las 13:00 h el aSSP p-Arbust-Arbor con la pradera sin árboles. Esta reducción, representó una disminución en 27,4\% de FR para los animales que pastorearon el aSSP. Barragán-Hernández et al. (2015), en una evaluación del efecto del estrés calórico en vacas F1 Holsteín x Cebú y trihibridas Romosinuano, Holsteín y Cebú, bajo los mismos aSSP, registraron una disminución de la FR del orden de 21,5\% al comparar los registros del aSSP p-Arbust-Arbor, con la pradera sin árboles.

Los animales Romosinuanos, pese a ser considerados como una raza adaptada a las condiciones tropicales (Ossa-Saraz et al., 2013), presentaron una mayor tasa respiratoria cuando fueron sometidos a condiciones ambientales adversas, comparados con los animales de la raza Cebú Brahman. Autores como Hammond et al. (1996), han considerado el Romosinuano como una raza taurina termotolerante, con características particulares para soportar condiciones de calor como lo son: piel suelta, pelo corto y baja tasa metabólica (Scharf et al., 2010). Sin embargo, Hammond et al. (1996) al comparar las respuestas fisiológicas al estrés por calor de razas taurinas e índicas, evidenció que a un ITH de 85, hembras de la raza Romosinuano exhibieron una mayor FR que las Brahman (56 $\pm 2,1$ vs 36 $\pm 2,4 \mathrm{rpm}$, respectivamente), asimismo, Cardozo et al. (2015) evidenciaron que bajo un ITH de 78, animales Romosinuano presentaron una FR 9,3 rpm más que los animales Cebú Brahman, lo que indica un grado de estrés leve. Se ha demostrado en animales Guzerat (cebuino) que al pasar de $24{ }^{\circ} \mathrm{C}$ a $35^{\circ} \mathrm{C}$ solo se incrementan dos respiraciones por minuto, con un volumen de inspiración de 2,2 $1 \mathrm{~s}^{-1}$ (Camerro et al., 2016), lo cual corrobora las ventajas evolutivas que poseen los animales cebuinos para enfrentar ambientes calurosos, con una respiración lenta y profunda, habilitada por un gran paquete celular que le permite acarrear mayor cantidad de oxígeno, baja tasa metabólica y mayor cantidad y distribución de glándulas sudoríparas (Hansen, 2004; Scharf et al., 2010).

Con relación a los dípteros hematófagos, se ha documentado que el umbral de daño económico causado puede variar desde veinticinco moscas en más del $60 \%$ de los animales para Stomoxys calcitrans (Giraldo et al., 2011), hasta cien en el caso de Haematobia irritans (Benavides, 2010). En la presente investigación, solo se reportaron poblaciones por encima de 150 dípteros por animal en el tratamiento Pasto para el mes de mayo, y las poblaciones más bajas en el aSSP p-Arbusto, con una tendencia decreciente a través del tiempo.

En los aSSP se ha reportado que especies competidoras por el sustrato para la reproducción de los dípteros, actúan como importantes controladores biológicos de estos parásitos (Giraldo et al., 2011). En este grupo, se destacan los escarabajos coprófagos (Coleoptera: Scarabaeidae: Scarabaeinae), por poseer la capacidad de remover con gran eficiencia las bostas de los bovinos, las cuales han sido identificadas como un aspecto clave dentro del ciclo biológico de moscas hematófagas (Giraldo-Echeverri et al., 2018).

En un estudio sobre estructura y diversidad de escarabajos coprófagos en los aSSP objeto de esta investigación, Murillo-Ramos et al. (2009) identificaron que el aSSP p-Arbusto registró la mayor abundancia con 1880 individuos ha $^{-1}$, lo que superó a los tratamientos Pasto y p-Arbut-Arbor en 7 y $28 \%$, respectivamente, dentro de las especies encontradas se destacaron los géneros Dichotomius, Ontophagus y Diabroctis, descritos como importantes removedores de estiércol (Giraldo-Echeverri et al., 2018).

En una evaluación de la infestación por ecto y endoparásitos para la raza Nelore bajo sistemas silvopastoriles conformados por Eucalyptus globulus y Urochloa brizantha, Gaudio et al. (2018) reportaron menos infestación por dípteros hematófagos, evaluada en una escala de 1 (menor infestación) a 5 (mayor infestación), para el aSSP versus sistemas convencionales sin sombra (1 vs 4 respectivamente). De igual forma, en aSSP con Acacia mangium, Gmelina arborea, Piptadenia peregrina y Brachiaria decumbens con animales de la raza Romosinuano, Benavides-Cruz (2013) registró 41 moscas por animal, contra 86 obtenidas en el monocultivo de $B$. decumbens. 


\section{Conclusiones}

Los arreglos silvopastoriles (aSSP) evaluados demostraron ventajas comparativas para minimizar el impacto del estrés calórico en animales de la raza Romosinuano y Cebú Brahman, en particular el aSSP p-Arbust-Arbor, mantuvo la frecuencia respiratoria de los animales dentro de los rangos descritos para considerar termoneutralidad.

Los animales que pastorearon el aSSP p-Arbust, presentaron menor conteo de dípteros hematófagos, lo cual pudo estar influenciado por una posible participación de organismos que compiten por espacios de ovoposición requeridos por los dípteros hematófagos. Esto sugiere que estos esquemas de producción pueden contribuir a la reducción en el uso de productos químicos para el control de ectoparásitos.

\section{Agradecimientos}

Los autores agradecen a la Corporación Colombiana de Investigación Agropecuaria (AGROSAVIa), por el soporte financiero y logístico para realizar el presente trabajo.

\section{Literatura citada}

Ahlberg, C.M., K. Allwardt, A. Broocks, K. Bruno, L. McPhillips, A. Taylor, C.R. Krehbiel, M.S. Calvo-Lorenzo, C.J. Richards, S.E. Place, U. DeSilva, D.L. VanOverbeke, R.G. Mateescu, L.A. Kuehn, R.L. Weaber, J.M. Bormann, and M.M. Rolf. 2018. Environmental effects on water intake and water intake prediction in growing beef cattle. J. Anim. Sci. 96:43684384. doi:10.1093/jas/sky267

Alegría-López, M.A., R.I. Rodríguez-Vivas, J.F.J. Torres-Acosta, M.M. Ojeda-Chi, and J.A. Rosado-Aguilar. 2015. Use of ivermectin as endoparasiticide in tropical cattle herds generates resistance in gastrointestinal nematodes and the tick Rhipicephalus microplus (Acari: Ixodidae). J. Med. Entomol. 52:214-221. doi:10.1093/jme/tju025

Alonso-Díaz, M.Á., J.F.J. Torres-Acosta, C.A. Sandoval-Castro, and W.B. Campbell. 2014. Sustainable food production includes human and environmental health. In: W.B. Campbell, and S. López-Ortíz, editors, Issues in Agroecology - Present status and future prospectus. Vol. 3. Springer, Dordrecht, NLD. p. 191-228. doi:10.1007/978-94-007-7454-4

Arias, R.A., T.L. Mader, y P.C. Escobar. 2008. Factores climáticos que afectan el desempeño productivo del ganado bovino de carne y leche. Arch. Med. Vet. 40:7-22. doi:10.4067/S0301-732X2008000100002

Atkins, I., M. Cook, and C. Choi. 2018. Continuous respiration rate measurement of heat-stressed dairy cows and relations to environment, body temperature, and lying time. Trans. ASABE 61:1475-1485. doi:10.13031/trans.12451

Barragán-Hernández, W.A., L. Mahecha-Ledesma, y Y.S. Cajas-Girón. 2015. Variables fisiológicas-metabólicas de estrés calórico en vacas bajo silvopastoreo y pradera sin árboles. Agron. Mesoam. 26:211-223. doi:10.15517/am.v26i2.19277

Bates, D., M. Mächler, B.M. Bolker, and S.C. Walker. 2015. Fitting linear mixed-effects models using lme4. J. Stat. Softw. 67:1-48. doi:10.18637/jss.v067.i01

Benavides, E. 2010. Manejo integrado de los parásitos (II). Carta Fedegán 117:16-21.

Benavides-Cruz, J.C. 2013. Influencia de moscas hematófagas de bovinos sobre la ganancia de peso y se relacion con algunas variables climáticas bajo dos sistemas de pastores (tradicional y silvopastoril) en el Centro de Investigación La Libertad de CORPOICA Villavicencio Meta. Tesis M.Sc., CORPOICA, Bogotá, COL. 
Brooks, M.E., K. Kristensen, K.J. van-Benthem, A. Magnusson, C.W. Berg, A. Nielsen, H.J. Skaug, M. Mächler, and B.M Bolker. 2017. glmmTMB balances speed and flexibility among packages for zero-inflated generalized linear mixed modeling. The R J. 9:378-400.

Broom, D.M. 2017. Components of sustainable animal production and the use of silvopastoral systems. Rev. Bras. Zootec. 46:683-688. doi:10.1590/S1806-92902017000800009

Cajas-Giron, Y.S., and F.L. Sinclair. 2001. Characterization of multistrata silvopastoral systems on seasonally dry pastures in the Caribbean Region of Colombia. Agrofor. Syst. 53:215-225. doi:10.1023/A:1013384706085

Camerro, L.Z., A.S. Maia, M.C. Neto, C.C. Costa, and P.A. Castro. 2016. Thermal equilibrium responses in Guzerat cattle raised under tropical conditions. J. Therm. Biol. 60:213-221. doi:10.1016/j.jtherbio.2016.07.007

Cardozo, J.A., J. Gallego, J. Tobón, W. Barragán, N. Restrepo, D. Bejarano, y C. Lucero. 2015. Efecto de variaciones climáticas en el comportamiento de los signos vitales, hormonas tiroideas y proteínas totales de bovinos Cebú y criollos bon, romo y costeño con cuernos. En: G. Martínez et al., editores, Libro de resúmenes del XVI Simposio sobre conservación y utilización de recursos zoogenéticos. Asociación de Criadores de Ganado Criollo de los Llanos Orientales, Red CONBIAND Colombia, y Universidad Nacional de Colombia, Villavicencio, COL. p. 115.

Castro, E., A. Gil, M.A. Solari, and N.A. Farias. 2005. Validation of a subjective counting method for a horn flies (Haematobia irritans irritans) (Diptera: Muscidae) population in a cattle herd. Vet. Parasitol. 133:363-367. doi:10.1016/j. vetpar.2005.06.003

Cayuela, L. 2012. Modelos lineales mixtos (LMM) y modelos lineales generalizados mixtos (GLMM) en R. ORANGEDOX, Vancouver, CAN. https://dl.orangedox.com/XpAzAQ0duvnoBmWZJ2/5-Modelos\%20lineales\%20mixtos\%20en\%20R. pdf (consultado 21 feb. 2019).

Chang-Fung-Martel, J., M.T. Harrison, R. Rawnsley, A.P. Smith, and H. Meinke. 2017. The impact of extreme climatic events on pasture-based dairy systems: A review. Crop Past. Sci. 68:1158-1169. doi:10.1071/CP16394

Collier, R.J., R.B. Zimbelman, R.P. Rhoads, M.L Rhoads, and L.H. Baumgard. 2011. A Re-evaluation of the impact of temperature humidity index (THI) and black globe humidity index (BGHI) on milk production in high producing dairy cows. In: M. Gamroth, editor, Proceedings of the Western Dairy Management Conference. Western Dairy Management Conference, Reno, NV, USA. p. 113-126.

Di-Rienzo, J.A., R. Macchiavelli, y F. Casanoves. 2009. Modelos mixtos en InfoStat. Universidad de Puerto Rico, PUR. http:// academic.uprm.edu/rmacchia/agro6998/TutorialMMesp.pdf (consultado 4 may. 2018).

Dikmen, S., and P.J. Hanse. 2009. Is the temperature-humidity index the best indicator of heat stress in lactating dairy cows in a subtropical environment? J. Dairy Sci. 92:109-116. doi:10.3168/jds.2008-1370

Galán, E., P. Llonch, A. Villagrá, H. Levit, S. Pinto, and A. del-Prado. 2018. A systematic review of non-productivity-related animal-based indicators of heat stress resilience in dairy cattle. PLoS One 13(11):e0206520. doi:10.1371/journal. pone. 0206520

Gaudio, N.A.R., B.B. Cardoso, G.M. Spessotto, J.P. Luperini, B.E. Anacleto, D.P. da-Silva, M.A. Calado, e M.A. Factori. 2018. Avaliação de endoparasitas e ectoparasitas em bovinos da raça Nelore em sistema silvipastoril e convencional, suplementados ou não. Adaltech, BRA. BRA. http://www.adaltech.com.br/anais/zootecnia2018/resumos/trab-0189.pdf (consultado 21 feb. 2019).

Gaughan, J., S.M. Holt, G.L. Hahn, T. Mader, and R.A. Eigenberg. 2000. Respiration rate - Is it a good measure of heat stress in cattle? Asian-Aus. J. Anim. Sci. 13:329-332. 
Giraldo, C., L.K. Reyes, y J. Molina. 2011. Manejo integrado de artrópodos y parásitos en sistemas silvopastoriles intensivos. Manual 2, Proyecto ganadería colombiana sostenible. Banco Mundial, FEDEGAN, CIPAV, Fondo Acción, y TNC, Bogotá, COL.

Giraldo-Echeverri, C., S. Montoya-Molina, y F. Escobar. 2018. Escarabajos del estiércol en paisajes ganaderos de Colombia. CIPAV, Cali, COL.

Grace, D., B. Bett, J. Lindahl, and T. Robinson. 2015. Climate and livestock disease: assessing the vulnerability of agricultural systems to livestock pests under climate change scenarios. Working Paper no. 116. CCAFS, Copenhagen, DNK.

Hammond, A.C., T.A. Olson, C.C. Chase, E.J. Bowers, R.D. Randel, C.N. Murphy, D.W. Vogt, and A. Tewolde. 1996. Heat tolerance in two tropically adapted Bos taurus breeds, Senepol and Romosinuano, compared with Brahman, Angus, and Hereford cattle in Florida. J. Anim. Sci. 74:295-303. doi:10.2527/1996.742295x

Hansen, P.J. 2004. Physiological and cellular adaptations of zebu cattle to thermal stress. Anim. Reprod. Sci. 82-83:349-360. doi:10.1016/j.anireprosci.2004.04.011

Herrero, M., P. Havlik, J. McIntire, A. Palazzo, and H. Valin. 2014. African livestock futures: Realizing the potential of livestock for food security, poverty reduction and the environment in Sub-Saharan Africa. Office of the Special Representative of the UN Secretary General for Food Security and Nutrition and the United Nations System In-fluenza Coordination (UNSIC), Geneva, CHE.

Holdridge, L.R. 1971. Forest environments in tropical life zones: A pilot study. Pergamon Press, Oxford, GBR.

Kadzere, C.T., M.R. Murphy, N. Silanikove, and E. Maltz. 2002. Heat stress in lactating dairy cows: A review. Livest. Prod. Sci. 77:59-91. doi:10.1016/S0301-6226(01)00330-X

Lallo, C.H.O., J. Cohen, D. Rankine, M. Taylor, J. Cambell, and T. Stephenson. 2018. Characterizing heat stress on livestock using the temperature humidity index (THI)-prospects for a warmer Caribbean. Reg. Environ. Change 18:2329-2340. doi:10.1007/s10113-018-1359-x

Lima, L.G.F., S.H.V. Perri, and A.P. Prado. 2003. Variation in population density of horn flies (Haematobia irritans irritans) (L.) (Diptera: Muscidae) in Nellore cattle (Bos indicus). Vet. Parasitol. 117:309-314. doi:10.1016/j.vetpar.2003.10.002

Lopes, L.B., C. Eckstein, D.S. Pina, and R.A. Carnevalli. 2016. The influence of trees on the thermal environment and behaviour of grazing heifers in Brazilian Midwest. Trop. Anim. Health Prod. 48:755-761. doi:10.1007/s11250-016-1021-x

Molina-Benavides, R.A., H. Sánchez-Guerrero, and A. Stanislao-Atzori. 2018. A conceptual model to describe heat stress in dairy cows from actual to questionable loops. Acta Agron. 67:59-64. doi:10.15446/acag.v67n1.60612

Murgueitio-Restrepo, E., R. Barahona-Rosales, M.X. Flores-Estrada, J.D. Chará-Orozco, y J.E. Rivera-Herrera. 2016. Es posible enfrentar el cambio climático y producir más leche y carne con sistemas silvopastoriles intensivos. Ceiba 54:2330. doi:10.5377/ceiba.v54i1.2774

Murillo-Ramos, L., J.C. Linares, e Y.S. Cajas. 2009. Estructura de la comunidad de coleópteros coprófagos (Scarabaeinae: Aphodiinae) en sistemas silvopastoriles de estratos múltiples, Corpoica C.I. Turipaná. Tesis Lic., Universidad de Córdoba, COL.

Nardone, A., B. Ronchi, N. Lacetera, M.S. Ranieri, and U. Bernabucci. 2010. Effects of climate changes on animal production and sustainability of livestock systems. Livest. Sci. 130:57-69. doi:10.1016/j.livsci.2010.02.011

Oliveira, R., M. Faria, R. Silva, L. Bezerra, G. Carvalho, A. Pinheiro, J. Simionato, and A. Leão. 2015. Fatty acid profile of milk and cheese from dairy cows supplemented a diet with palm kernel cake. Molecules 20:15434-15448. doi:10.3390/ molecules200815434 
Oliveira, C., F. Villa, R. Almeida E. Gamarra, S. Villela, and P. Martins. 2017. Thermal comfort indices assessed in integrated production systems in the Brazilian savannah. Agrofor. Syst. 92:1659-1672. doi:10.1007/s10457-017-0114-5

Ossa-Saraz, G., A. David-Hinestroza, M. Santana-Rodríguez, S. Reza-García, J. Pérez-García, y Y. Abuabara-Pérez. 2013. Formación, desarrollo y caracterización fenotípica de los caracteres productivos y reproductivos del hato Romosinuano del banco de germoplasma de Colombia. Cienc. Tecnol.Agropecu. 14(2):231-243.doi:10.21930/rcta.vol14_num2_art:503

Pankaj, P.K., D.B.V. Ramana, R. Pourouchottamane, and S. Naskar. 2013. Livestock management under changing climate scenario in India. World J. Vet. Sci. 1:25-32. doi:10.12970/2310-0796.2013.01.01.5

Pérez, E., M. Soca, L. Díaz, y M. Corzo. 2008. Comportamiento etológico de bovinos en sistemas silvopastoriles en Chiapas, México. Pastos y Forrajes 31(2):161-172.

Pulido, M., L. Ramírez-Avilés, F.J. Solorio, I. Fiebrig, and S. Burbi. 2018. The impact of veterinary medicine and animal husbandry on the biophysical characteristics of soils in neotropical agroecosystems. Soil Syst. 2(2):24. doi:10.3390/ soilsystems 2020024

Rao, I., M. Peters, A. Castro, R. Schultze-Kraft, D. White, M. Fisher, J. Miles, C. Lascano, M. Blümmel, D. Bungenstab, J. Tapasco, G. Hyman, A. Bolliger, B. Paul, R. van der Hoek, B. Maass, T. Tiemann, M. Cuchillo, S. Douxchamps, C. Villanueva, A. Rincón, M. Ayarza, T. Rosenstock, G. Subbarao, J. Arango, J. Cardoso, M. Worthington, N. Chirinda, A. Notenbaert, A. Jenet, A. Schmidt, N. Vivas, R. Lefroy, K. Fahrney, E. Guimarães, J. Tohme, S. Cook, M. Herrero, M. Chacón, T. Searchinger, and T. Rudel. 2015. LivestockPlus - The sustainable intensification of forage-based agricultural systems to improve livelihoods and ecosystem services in the tropics. Trop. Grass1. 3(2):59-82. doi:10.17138/TGFT(3)5982

Robertshaw, D. 2006. Mechanisms for the control of respiratory evaporative heat loss in panting animals. J. Appl. Physiol. 101:664-668. doi:10.1152/japplphysiol.01380.2005

Scharf, B., J.A. Carroll, D.G. Riley, C.C. Chase, S.W. Coleman, D.H. Keisler, R.L. Weaber, and D.E. Spiers. 2010. Evaluation of physiological and blood serum differences in heat-tolerant (Romosinuano) and heat-susceptible (Angus) Bos taurus cattle during controlled heat challenge. J. Anim. Sci. 88:2321-2336. doi:10.2527/jas.2009-2551

Schütz, K.E., N.R. Cox, and L.R. Matthews. 2008. How important is shade to dairy cattle? Choice between shade or lying following different levels of lying deprivation. Appl. Anim. Behav. Sci. 114:307-318. doi:10.1016/j.applanim.2008.04.001

Tucker, C.B., A.R. Rogers, and K.E. Schütz. 2008. Effect of solar radiation on dairy cattle behaviour, use of shade and body temperature in a pasture-based system. Appl. Anim. Behav. Sci. 109:141-154. doi:10.1016/j.applanim.2007.03.015

Weindl, I., H. Lotze-Campen, A. Popp, C. Müller, P. Havlík, M. Herrero, C. Schmitz, and S. Rolinski. 2015. Livestock in a changing climate: production system transitions as an adaptation strategy for agriculture. Environ. Res. Lett. 10(9):094021. doi:10.1088/1748-9326/10/9/094021

Zuur, A.F., E.N.I. Ieno, N.J. Walker, A.A. Saveliev, and G.M. Smith. 2009. Mixed effects models and extensions in ecology with R. Springer-Verlag, NY, USA. 